
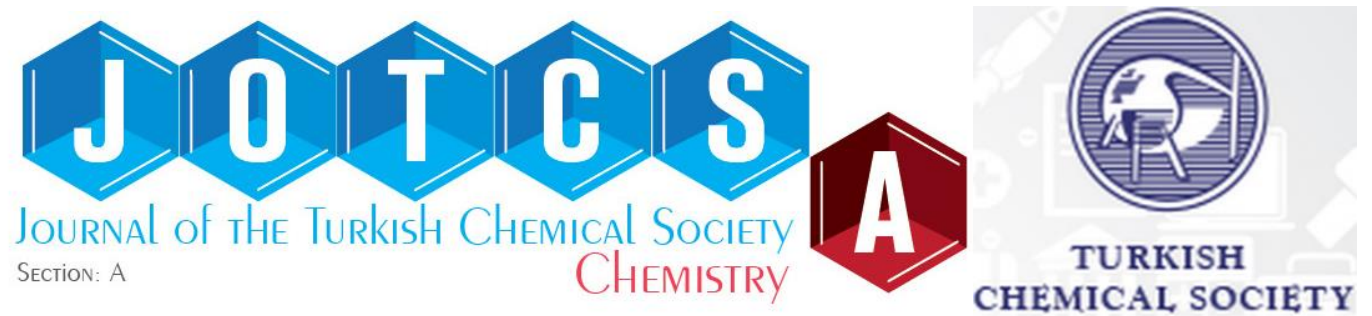

\title{
A Novel One-Pot Green Synthesis and Characterization of 5- Substituted Bis-Iminothiazolidinones
}

\author{
Fatma Tülay Tuğcu1* \\ ${ }^{1}$ Yildiz Technical University, Faculty of Arts and Sciences, Department of Chemistry, (34220), İstanbul, \\ Turkey.
}

Abstract: In this study, some new 5-substituted bis-iminothiazolidinone derivatives have been synthesized via one-pot green synthesis. The study comprises two steps. In the first step, bis-thioureas were prepared by the reaction of aryl isothiocyanates with substituted amines. In the second step, substituted bisthioureas prepared beforehand and chloroacetic acid were condensed with substituted thiophene-2carboxaldehydes and new 5-substituted bis-iminothiazolidinone compounds were obtained. The structures of all these synthesized compounds were determined and characterized by infrared, nuclear magnetic resonance and mass spectral data.

Keywords: 5-Substituted bis-iminothiazolidinone, bis-thiourea, one-pot reaction, green synthesis.

Submitted: July 03, 2018. Accepted: September 04, 2018.

Cite this: Tuğcu F. A Novel One-Pot Green Synthesis and Characterization of 5-Substituted BisIminothiazolidinones. JOTCSA. 2018;5(3):1061-70.

DOI: $\underline{\text { http://dx.doi.org/10.18596/jotcsa.440080. }}$

*Corresponding author. E-mail: ttugcu@yahoo.com.

\section{INTRODUCTION}

One-pot method without catalyst and solvent saves both time and energy since many intermediate products and final products do not need to be purified (1-2). Also, due to the growing concern for the influence of organic solvents on the environment as well as on the human body, development of solvent-free organic reactions have attracted the attention of synthetic organic chemists. These types of reactions are simple to handle, reduce pollution and comparatively cheaper to operate. For these reasons, solventless reaction complies with the fifth principle of green chemistry. It is believed that solvent-free synthesis is industrially useful and largely green. Green chemistry, also called sustainable chemistry, are processes that reduce or eliminate the use and generation of hazardous substances. From this point of view, one-pot solvent-free reactions are regarded as an ideal method of green synthesis (3).

Iminothiazolidinones have attracted much interest over the years since they are important and versatile scaffolds having engaged a pronounced position in medicinal chemistry. They are well-known to possess an extensive spectrum of biological activities (4-10). There are many existing methods for the synthesis of 5substituted iminothiazolidinones, but they are generally synthesized in two steps (11-15). In the first step, iminothiazolidinones are formed by the reaction of thioureas or $\mathrm{N}$-substituted thioureas with a-chloroacetic acid or their acid esters or amides. In the second step, these compounds are reacted with aromatic aldehydes in basic medium to give 5-substituted iminothiazolidinone compounds. For example, Ottana et al. (12) synthesized the 2-imino-4-thiazolidinone compound as a result of the reaction of $\mathrm{N}$-propylN'-phenylthiourea compound prepared from phenylisothiocyanate and propylamine with chloroacetyl chloride in triethylamine medium; this compound was then condensed with aromatic aldehydes in piperidine-containing medium to give 5-arylidene-2-imino-4-thiazolidinones.

There are few articles in the literature for bisiminotiyazolidinone (16) despite the many publications on the synthesis of 5-substituted iminotiazolidinone compounds. Therefore, in this article, reports the synthesis of new 5-substituted bis-iminothiazolidinone derivatives by the one- 
pot solvent free green reaction from the corresponding substituted thiophene-2carbaldehyde with bis-thiourea and chloroacetic acid at $70^{\circ} \mathrm{C}$. This article is consistent with the green chemistry approach because it describes a reaction which take place at low temperature and does not use solvents and catalysts, making it environmentally friendly, efficient and economic.

\section{MATERIALS AND METHODS}

Reagents purchased from Merck were as follows: dichloromethane, chloroform, ethyl acetate, nhexane, chloroacetic acid, thiophene-2carboxaldehyde, 3-methylthiophene-2carboxaldehyde, phenyl isothiocyanate, p-tolyl isothiocyanate, 1,4-phenylenediamine, 4,4'methylenedianiline, silica gel 60 (0.063-0.200 $\mathrm{mm}$ ), and sea sand. All reagents were used as purchased from the manufacturer. Dichloromethane, chloroform, and n-hexane were used after purification for column chromatography. TLC was carried out on aluminum sheets pre-coated with silica gel 60 F254 purchased from Merck, and the spots were visualized with UV light (254/366 nm) using a Camag UV lamp.

NMR $\left({ }^{1} \mathrm{H}\right.$ and $\left.{ }^{13} \mathrm{C}\right)$ spectra were saved on a Bruker $500 \mathrm{MHz}$ NMR spectrometer at Yildiz Technical University and Erzurum Atatürk University, Department of Chemistry. $\mathrm{CDCl}_{3}$ was used as a solvent. FTIR spectra were recorded on a Philips PU 9714 ATR spectrometer using a Perkin-Elmer Spectrum One program at Yildiz Technical University, Instrumental Analysis Laboratory. Melting points were obtained with a Gallenkamp Melting Point Apparatus in open capillaries with no correction.

\section{Preparation of Bis-thiourea Derivatives (1a-} d)

A mixture of the appropriate diamine $(1 \mathrm{mmol})$ and substituted phenyl isothiocyanate $(2.4 \mathrm{mmol})$ was stirred in $\mathrm{CH}_{2} \mathrm{Cl}_{2}$ at room temperature for 24 hours. The crude product was concentrated under vacuum and recrystallized from ethanol. General synthesis of bis-thioureas is summarized in Figure 1 .

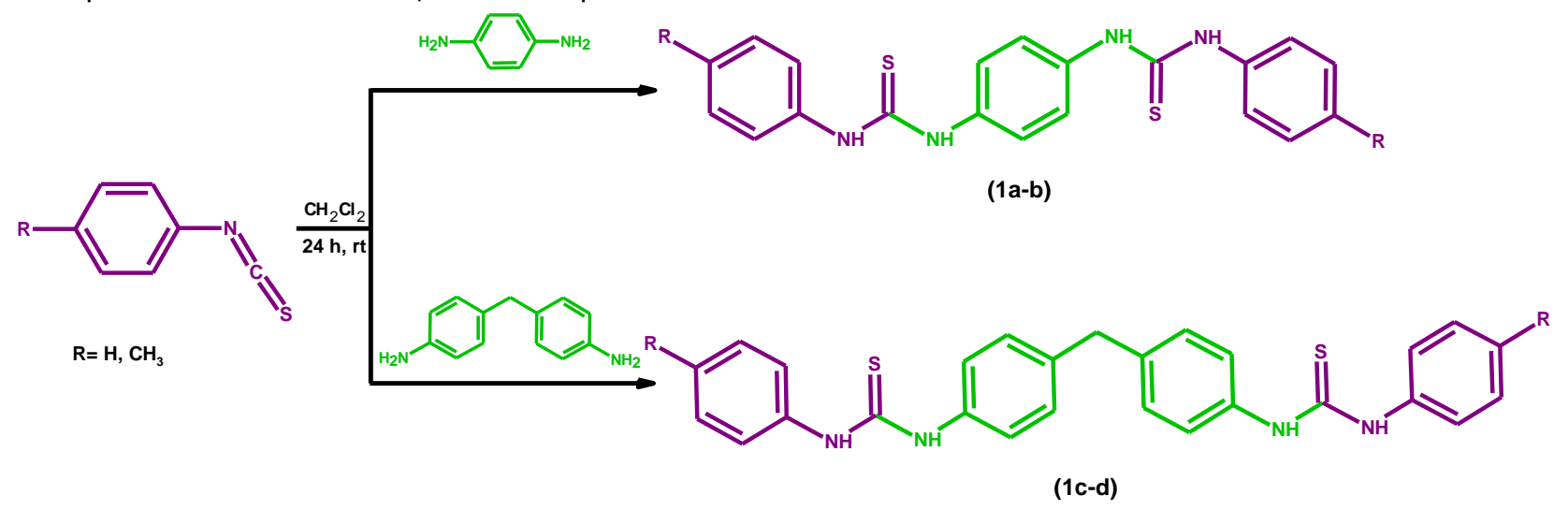

Figure 1. Synthesis of bis-thiourea derivatives.

\begin{abstract}
Preparation of 5-Substituted BisIminothiazolidinone Derivatives ( $2 a-h)$ To synthesize 5-substituted bisiminothiazolidinone compounds, the appropriate bis-thiourea (1 $\mathrm{mmol})$, chloroacetic acid (2.4 $\mathrm{mmol}$ ) and substituted thiophene-2-carbaldehyde
\end{abstract}

( $2 \mathrm{mmol}$ ) were stirred at room temperature for 4 hours and then heated at $70{ }^{\circ} \mathrm{C}$ for 6 hours. The product was purified by column chromatography. General synthesis of these compounds is summarized in Figure 2. 


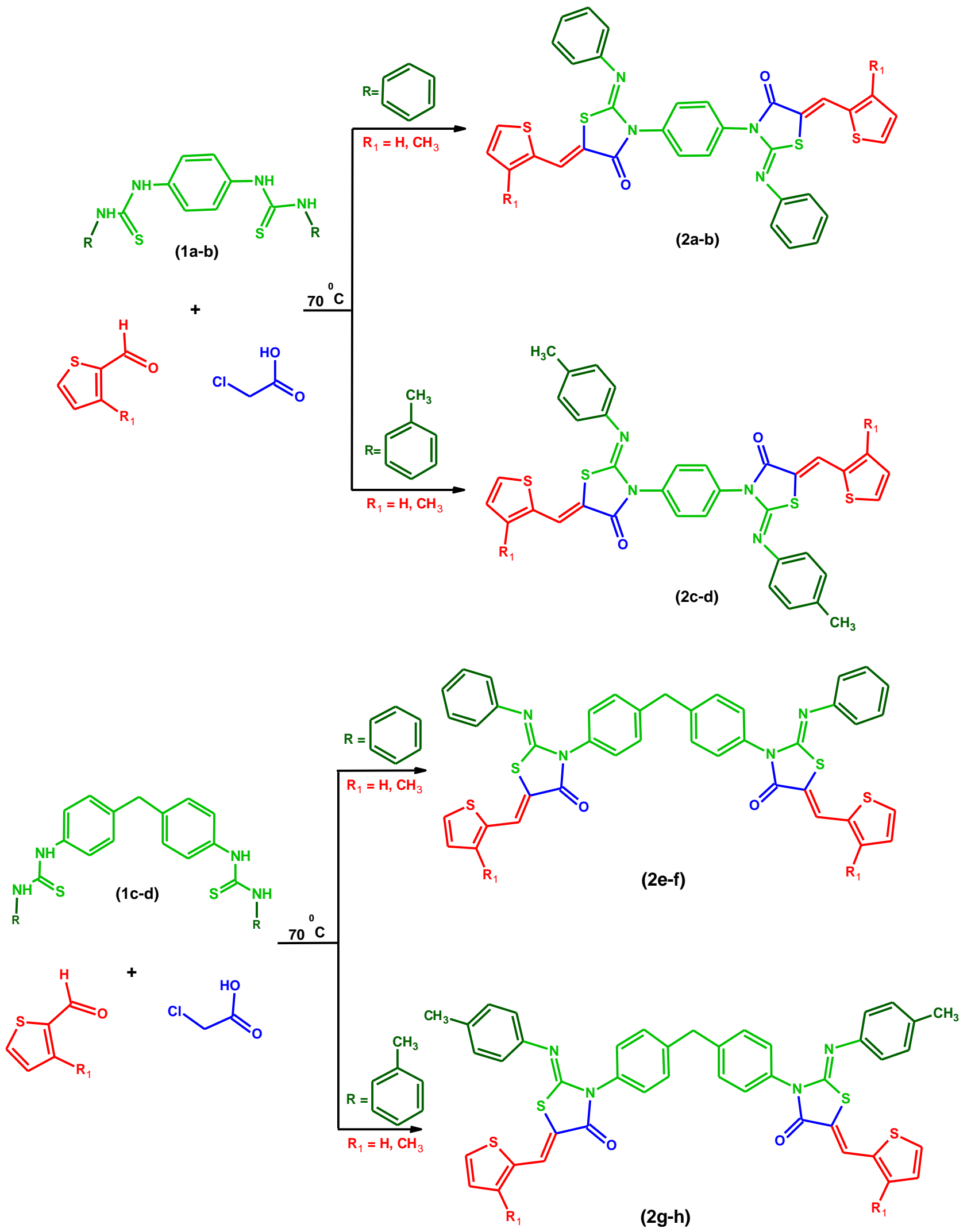

Figure 2. Synthesis of 5-substituted bis-iminothiazolidinone derivatives.

\section{RESULTS AND DISCUSSION}

The synthesis of bis-thiourea (1a-d) was done using diamine and substituted phenyl isothiocyanate in $\mathrm{CH}_{2} \mathrm{Cl}_{2}$ at room temperature.
They were recrystallized from hot ethanol and were characterized by recording their spectral data (Table 1). A mechanism for the reaction of bis-thiourea is shown in Figure 3 (17). 


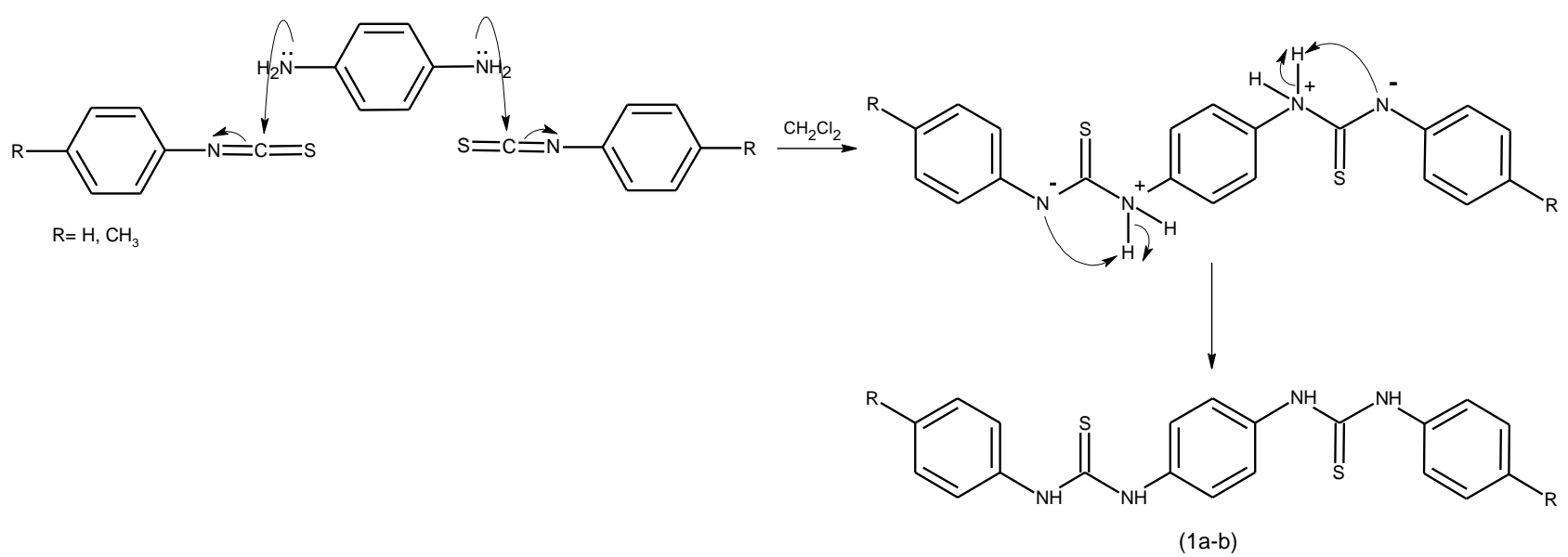

Figure 3. Proposed mechanism for the formation of bis-thiourea derivatives.

When the FTIR spectrum of bis-thioureas (1a-d) were analyzed, the conjugated $\mathrm{C}=\mathrm{C}$ and $\mathrm{C}-\mathrm{N}$ vibrations were observed in the ranges of $1600-$ 1550 and $1540-1490 \mathrm{~cm}^{-1}$, respectively. On the other hand, the $\mathrm{N}-\mathrm{H}$ vibrations (3467-3438 and $3315-3360 \mathrm{~cm}^{-1}$ ) of diamine used as reagents and the $\mathrm{N}=\mathrm{C}=\mathrm{S}$ stretching bands $\left(2200-2000 \mathrm{~cm}^{-1}\right)$ of substituted isothiocyanate disappeared, which indicates that condensation had taken place.

Eight new 5-substituted bis-iminothiazolidinone derivatives $(\mathbf{2} \mathbf{a}-\mathbf{h})$ were obtained from the second step of the present study. The synthesized crude products were obtained in varying yield depending on the structure and were purified by column chromatography. For the one-pot condensation method between bis-thiourea, thiophene-2-carboxaldehyde and chloroacetic acid, an estimated mechanism for the formation of 5-substituted bis-iminothiazolidinone was proposed by referring to the literature in Figure 4 (18-19). The structures of all synthesized compounds ( $\mathbf{2} \mathbf{a}-\mathbf{h})$ were explained based on the analysis of their spectroscopic data (Table 1 ).

When the infrared spectra of 5-substituted bisiminothiazolidinones were analyzed, the conjugated $\mathrm{C}=\mathrm{C}$ and $\mathrm{C}=\mathrm{N}$ stretching vibrations that were distinctive for nitrogen having heterocyclic compounds are observed in the ranges of $1600-1500$ and $1670-1600 \mathrm{~cm}^{-1}$, respectively. Moreover, the $\mathrm{N}-\mathrm{H}$ vibrations (3295$3190 \mathrm{~cm}^{-1}$ ) of bis-thiourea, the $\mathrm{C}=\mathrm{O}$ stretching $\left(\sim 1674 \mathrm{~cm}^{-1}\right)$ of heteroaromatic aldehyde and the -OH vibration bands $\left(3400-2800 \mathrm{~cm}^{-1}\right)$ of chloroacetic acid disappeared, which indicates that cyclization and condensation reactions occurred. The characteristic absorption bands of $\mathrm{C}=\mathrm{O}$ groups were observed at $1680-1722 \mathrm{~cm}^{-1}$ in the FTIR spectrum of the 5-substituted bisiminothiazolidinone derivatives.

In the second step of clarifying the structures, when the ${ }^{1} \mathrm{H}$ NMR spectra of the initial substances and those of the products were compared, the singlet pertaining to the - $\mathrm{CHO}$ group observed at around $11.0 \mathrm{ppm}$ in the spectra of substituted thiophene-2-carboxaldehydes and the singlet at around 9.8 and $9.3 \mathrm{ppm}$ belonging to the NHgroup in bis-thiourea were not encountered in ${ }^{1} \mathrm{H} \quad \mathrm{NMR}$ of the 5-substituted bisiminothiazolidinone (2a-h), which is an evidence of the suggested structures. The multiple peaks seen between 6.8-7.7 ppm in the lower area of the spectra show the proton resonances of aromatic and the hetero aromatic bond. The $\mathrm{H}$ atoms of $-\mathrm{CH}_{2}$ and $-\mathrm{CH}_{3}$ which belong to the compounds were observed in the ranges 4.03 ppm and 2.41-2.35 ppm respectively. 
Tuğcu F. JOTCSA. 2018;5(3): 1061-1070.<smiles>[R]NC(=S)Nc1ccc(NC(=S)NC(C)C(C)C)cc1</smiles><smiles>[R]N=C(Nc1ccc(NC(=N[R])SC([2H])([2H])C(=O)O)cc1)SC(C)C(=O)O</smiles><smiles>[R]N=C1SCC(O)(O)N1c1ccc(N2/C(=N/[R])SC([2H])C2(O)O)cc1</smiles><smiles>CO</smiles><smiles>[R]N=C1SC(C=CC=O)C(=O)N1c1ccc(N2C(=O)C(C=CC(=O)c3sccc3Br)SC2=N[R])cc1</smiles>

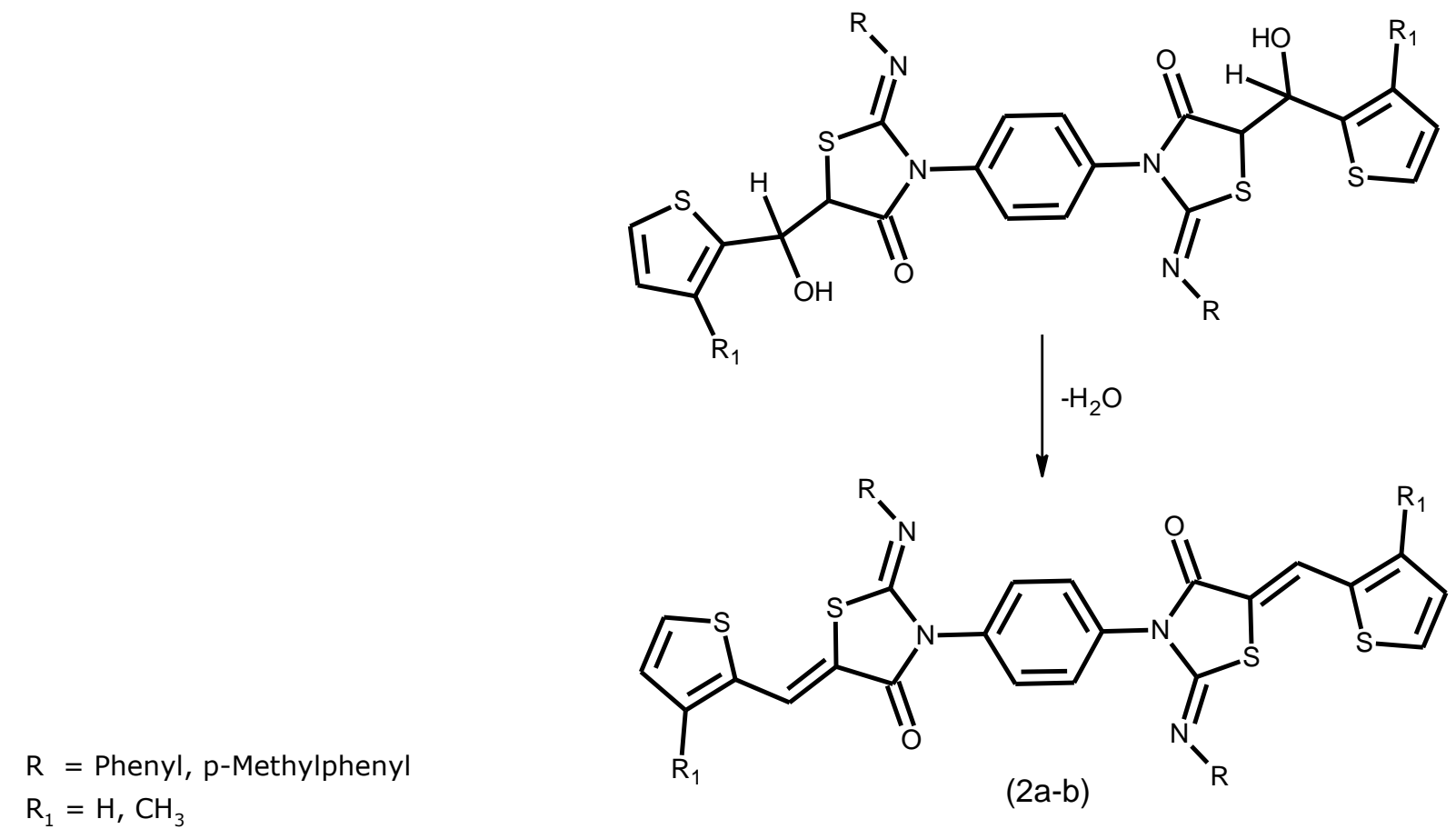

Figure 4. Proposed mechanism for the synthesis of 5 -substituted bis-iminothiazolidinones.

Mass spectra of the products were obtained in order to give certainty to the determined structure. When the mass spectra of compounds (2a-h) were examined, the observed molecular ion peaks confirmed the molecular weights of the products (Table 1 ). 
Tuğcu F. JOTCSA. 2018;5(3):1061-1070.

RESEARCH ARTICLE

1

Table 1. Physical, chemical, and spectral properties of all synthesized compounds.

\begin{tabular}{|c|c|c|c|c|c|}
\hline Compound & $\begin{array}{l}\text { Crystal } \\
\text { structure }\end{array}$ & $\begin{array}{l}\text { M.p } \\
\left({ }^{0} \mathrm{C}\right)\end{array}$ & $\begin{array}{l}\text { FTIR (ATR) } \\
\left(\gamma_{\left.\max / \mathrm{cm}^{-1}\right)}\right.\end{array}$ & $\begin{array}{c}{ }^{1} \mathrm{H} \text { NMR }(500 \mathrm{MHz}, d-\mathrm{DMSO}) \\
((\delta \mathrm{ppm})\end{array}$ & $\begin{array}{l}\mathrm{MS} \\
(\mathrm{m} / \mathrm{z})\end{array}$ \\
\hline $\begin{array}{c}\text { 1,1'-(1,4-Phenylene)bis(3-phenylthiourea) } \\
\text { (1a) (20) }\end{array}$ & White bar & $205-6$ & $\begin{array}{l}3221(\mathrm{~N}-\mathrm{H}), \\
2922(\mathrm{C}-\mathrm{H}), \\
1506(\mathrm{~N}-\mathrm{H}), \\
1072(\mathrm{C}-\mathrm{S}), \\
746(\mathrm{~N}-\mathrm{H})\end{array}$ & $\begin{array}{l}7.13(1 \mathrm{H}, \mathrm{t}), 7.34(2 \mathrm{H}, \mathrm{t}) \\
7.44(2 \mathrm{H}, \mathrm{s}), 7.49(2 \mathrm{H}, \mathrm{d}) \\
9.80(2 \mathrm{H}, \mathrm{s})\end{array}$ & \\
\hline $\begin{array}{c}1,1^{\prime} \text {-(1,4-Phenylene)bis(3-p-tolylthiourea) } \\
\text { (1b) (21) }\end{array}$ & White powder & $230-2$ & $\begin{array}{l}3295(\mathrm{~N}-\mathrm{H}), \\
2939(\mathrm{C}-\mathrm{H}), \\
1509(\mathrm{~N}-\mathrm{H}), \\
1141(\mathrm{C}-\mathrm{S}), \\
810(\mathrm{~N}-\mathrm{H})\end{array}$ & $\begin{array}{l}2.26(6 \mathrm{H}, \mathrm{s}), 6.52(2 \mathrm{H}, \mathrm{d}) \\
6.99(2 \mathrm{H}, \mathrm{d}), 7.10(2 \mathrm{H}, \mathrm{d}) \\
7.30(2 \mathrm{H}, \mathrm{d}), 9.31(2 \mathrm{H}, \mathrm{s})\end{array}$ & \\
\hline $\begin{array}{c}1,1^{\prime} \text { - }[4,4 \text { '-Methylenebis(1,4-phenylene) }] \text { bis(3-phenylthiourea) } \\
\text { (1c) (22) }\end{array}$ & White bar & $320-2$ & $\begin{array}{l}3190(\mathrm{~N}-\mathrm{H}), \\
2939(\mathrm{C}-\mathrm{H}), \\
1509(\mathrm{~N}-\mathrm{H}), \\
1233(\mathrm{C}-\mathrm{S}), \\
877(\mathrm{~N}-\mathrm{H})\end{array}$ & $\begin{array}{l}3.89(2 \mathrm{H}, \mathrm{s}), 7.12(1 \mathrm{H}, \mathrm{t}) \\
7.21(2 \mathrm{H}, \mathrm{d}), 7.32(2 \mathrm{H}, \mathrm{t}) \\
7.38(2 \mathrm{H}, \mathrm{d}), 7.47(2 \mathrm{H}, \mathrm{d}) \\
9.31(2 \mathrm{H}, \mathrm{s})\end{array}$ & \\
\hline $\begin{array}{l}\text { 1,1'-[4,4'-Methylenebis(1,4-phenylene) }] \text { bis(3-p-tolylthiourea) } \\
\text { (1d) }\end{array}$ & White powder & $230-1$ & $\begin{array}{l}3195(\mathrm{~N}-\mathrm{H}), \\
2920(\mathrm{C}-\mathrm{H}), \\
1586(\mathrm{~N}-\mathrm{H}), \\
1141(\mathrm{C}-\mathrm{S}), \\
820(\mathrm{~N}-\mathrm{H})\end{array}$ & $\begin{array}{l}2.51(6 \mathrm{H}, \mathrm{s}), 3.89(2 \mathrm{H}, \mathrm{s}) \\
7.21(4 \mathrm{H}, \mathrm{t}), 7.47(4 \mathrm{H}, \mathrm{d}) \\
9.31(2 \mathrm{H}, \mathrm{s})\end{array}$ & \\
\hline $\begin{array}{l}\text { 3,3'-(1,4-Phenylene)bis[2-(phenylimino)-5-(thiophen-2-ylmethylene) } \\
\text { thiazolidin-4-one }] \\
\mathbf{( 2 a )}\end{array}$ & $\begin{array}{l}\text { Light yellow } \\
\text { powder }\end{array}$ & $161-2$ & $\begin{array}{l}3015(=\mathrm{C}-\mathrm{H}), \\
1706(\mathrm{C}=\mathrm{O}), \\
1626(\mathrm{C}=\mathrm{N}), \\
1590(\mathrm{C}=\mathrm{C}), \\
1352(\mathrm{C}-\mathrm{N}), \\
827(\mathrm{C}-\mathrm{S})\end{array}$ & $\begin{array}{l}6.57(2 \mathrm{H}, \mathrm{d}, J=7.0 \mathrm{~Hz}, \mathrm{Ar}-\mathrm{H}), \\
6.70(2 \mathrm{H}, \mathrm{d}, J=7.4 \mathrm{~Hz}, A r-\mathrm{H}), \\
7.10(2 \mathrm{H}, \mathrm{d}, J=7.0 \mathrm{~Hz}, \mathrm{Ar}-\mathrm{H}), \\
7.18(1 \mathrm{H}, \mathrm{t}, J=7.4 \mathrm{~Hz}, \operatorname{Ar}-\mathrm{H}), \\
7.28(1 \mathrm{H}, \mathrm{t}, J=4.9 \mathrm{~Hz}, \operatorname{Ar}-\mathrm{H}), \\
7.40(2 \mathrm{H}, \mathrm{t}, J=8.1 \mathrm{~Hz}, \operatorname{Ar}-\mathrm{H}), \\
7.65(1 \mathrm{H}, \mathrm{d}, J=3.7 \mathrm{~Hz}, \operatorname{Ar}-\mathrm{H}), \\
7.90(1 \mathrm{H}, \mathrm{d}, J=5.1 \mathrm{~Hz}, \operatorname{Ar} \mathrm{H}), \\
8.05(1 \mathrm{H}, \mathrm{s}, \text { vinyl H) }\end{array}$ & $\begin{array}{l}647(M+1), \\
646\left(M^{+}\right), \\
645(M-1), \\
563, \\
479, \\
443, \\
361, \\
329\end{array}$ \\
\hline
\end{tabular}


3,3'-(1,4-Phenylene)bis $\{5-[(3-$ methylthiophen-2-yl)methylene $]-2-$ (phenylimino)thiazolidin-4-one\}

\section{(2b)}

3,3'-(1,4-Phenylene)bis[5-(thiophen-2-ylmethylene)-2-(p-tolylimino) thiazolidin-4-one]

(2c)

3,3'-(1,4-Phenylene)bis \{5-[(3-methylthiophen-2-yl)methylene]-2(p-tolylimino)thiazolidin-4-one $\}$

(2d)

3,3'-[4,4'-Methylene bis(4,1-phenylene)]bis[2-(phenylimino)5-(thiophen-2-ylmethylene)thiazolidin-4-one]

(2e)

$2.37\left(3 \mathrm{H}, \mathrm{s}, \mathrm{CH}_{3}\right)$,

$3082(=\mathrm{C}-\mathrm{H}), \quad 6.81(2 \mathrm{H}, \mathrm{d}, J=8.1 \mathrm{~Hz}, \mathrm{Ar}-\mathrm{H})$,

$2917\left(\mathrm{CH}_{3}\right), \quad 7.20(2 \mathrm{H}, \mathrm{d}, J=8.1 \mathrm{~Hz}, \mathrm{Ar}-\mathrm{H})$

$1704(\mathrm{C}=\mathrm{O}), \quad 7.29(1 \mathrm{H}, \mathrm{t}, \mathrm{J}=5.0 \mathrm{~Hz}, \mathrm{Ar}-\mathrm{H})$,

Light yellow

layer

181-2

$1624(\mathrm{C}=\mathrm{N}), \quad 7.38(2 \mathrm{H}, \mathrm{d}, \mathrm{J}=8.4 \mathrm{~Hz}, \mathrm{Ar}-\mathrm{H})$

$1595(\mathrm{C}=\mathrm{C}), \quad 7.41(2 \mathrm{H}, \mathrm{d}, \mathrm{J}=8.3 \mathrm{~Hz}, \mathrm{Ar}-\mathrm{H})$

$1352(\mathrm{C}-\mathrm{N})$,

$823(\mathrm{C}-\mathrm{S})$

$3.5 \mathrm{~Hz}, \mathrm{Ar}-\mathrm{H})$

$7.92(1 \mathrm{H}, \mathrm{d}, J=5.1 \mathrm{~Hz}, \operatorname{Ar}-\mathrm{H})$,

$8.05(1 \mathrm{H}, \mathrm{s}$, vinyl $\mathrm{H})$

$2.38\left(3 \mathrm{H}, \mathrm{s}, \mathrm{CH}_{3}\right)$

$3082(=\mathrm{C}-\mathrm{H}), \quad 6.60(2 \mathrm{H}, \mathrm{d}, J=7.0 \mathrm{~Hz}, \mathrm{Ar}-\mathrm{H})$,

$2917\left(\mathrm{CH}_{3}\right), \quad 6.95(2 \mathrm{H}, \mathrm{bd}, J=7.2 \mathrm{~Hz}, \mathrm{Ar}-\mathrm{H})$,

$1704(\mathrm{C}=\mathrm{O}), \quad 7.09(2 \mathrm{H}, \mathrm{d}, J=7.0 \mathrm{~Hz}, \mathrm{Ar}-\mathrm{H})$,

Light yellow

bar

173-4

$1585(\mathrm{C}=\mathrm{C}), \quad 7.18(1 \mathrm{H}, \mathrm{t}, \mathrm{J}=7.4 \mathrm{~Hz}, \mathrm{Ar}-\mathrm{H})$,

$1361(\mathrm{C}-\mathrm{N}), \quad 7.39(2 \mathrm{H}, \mathrm{t}, \mathrm{J}=8.2 \mathrm{~Hz}, \mathrm{Ar}-\mathrm{H})$,

827 (C-S) $7.82(1 \mathrm{H}, \mathrm{d}, J=5.1 \mathrm{~Hz}, \mathrm{Ar}-\mathrm{H})$,

$7.88(1 \mathrm{H}, \mathrm{s}$, vinyl $\mathrm{H})$

$2.40\left(3 \mathrm{H}, \mathrm{s}, \mathrm{CH}_{3}\right)$,

$3140(=\mathrm{C}-\mathrm{H}), \quad 2.49\left(3 \mathrm{H}, \mathrm{s}, \mathrm{CH}_{3}\right)$,

$2926(\mathrm{C}-\mathrm{H}), \quad 6.90(2 \mathrm{H}, \mathrm{d}, J=8.2 \mathrm{~Hz}, \mathrm{Ar}-\mathrm{H})$

$1722(\mathrm{C}=\mathrm{O}), \quad 7.13(1 \mathrm{H}, \mathrm{d}, J=4.9 \mathrm{~Hz}, \mathrm{Ar}-\mathrm{H})$

Light yellow

bar

186-7

$1601(\mathrm{C}=$

$1502(\mathrm{C}=\mathrm{C})$,

$1359(\mathrm{C}-\mathrm{N})$,

$7.20(2 \mathrm{H}, \mathrm{d}, J=8.2 \mathrm{~Hz}, \mathrm{Ar}-\mathrm{H})$

$7.35(2 \mathrm{H}, \mathrm{d}, J=8.6 \mathrm{~Hz}, \mathrm{Ar}-\mathrm{H})$,

,

$7.86(1 \mathrm{H}, \mathrm{d}, J=4.9 \mathrm{~Hz}, \mathrm{Ar}-\mathrm{H})$

$7.93(1 \mathrm{H}, \mathrm{s}$, vinyl $\mathrm{H})$

$3140(=\mathrm{C}-\mathrm{H}), \quad 4.03\left(2 \mathrm{H}, \mathrm{s}, \mathrm{CH}_{2}\right)$,

$2926(\mathrm{C}-\mathrm{H}), \quad 6.94(2 \mathrm{H}, \mathrm{d}, J=8.2 \mathrm{~Hz}, \mathrm{Ar}-\mathrm{H})$

$1682(\mathrm{C}=\mathrm{O}), \quad 6.99-7.17(3 \mathrm{H}, \mathrm{m}, \mathrm{Ar}-\mathrm{H})$

Light yellow powder
205-6
$7.21(2 \mathrm{H}, \mathrm{t}, \mathrm{J}=8.2 \mathrm{~Hz}, \mathrm{Ar}-\mathrm{H})$

$7.29(2 \mathrm{H}, \mathrm{m}, \mathrm{Ar}-\mathrm{H})$,

$1591(\mathrm{C}=\mathrm{C}), \quad 7.29(2 \mathrm{H}, \mathrm{m}, \mathrm{Ar}-\mathrm{H})$,

$\begin{array}{ll}1270(\mathrm{C}-\mathrm{N}), & 7.37-7.58(3 \mathrm{H}, \mathrm{m}, \mathrm{Ar} \\ 717(\mathrm{C}-\mathrm{S}) & 8.00(1 \mathrm{H}, \mathrm{s}, \text { vinyl } \mathrm{H})\end{array}$
$675(M+1)$,

$674\left(\mathrm{M}^{+}\right)$,

$673(M-1)$,

592 ,

508 ,

361 ,

329.

$675(M+1)$

$674\left(M^{+}\right)$,

$673(\mathrm{M}-1)$,

576 ,

480,

361

329

$704(M+1)$

$703\left(\mathrm{M}^{+}\right)$,

702 (M-1),

605,

508 ,

402 ,

361 ,

329

$738(M+1)$ $737\left(\mathrm{M}^{+}\right)$, $736(\mathrm{M}-1)$,

643 ,

569

375,

314 , 
3,3'-[4,4'-Methylenebis(4,1-phenylene)]bis $\{5-[(3-$ methylthiophen2-yl)methylene]-2-(phenylimino)thiazolidin-4-one $\}$

$$
\text { (2f) }
$$

3,3'-[4,4'-Methylenebis(4,1-phenylene)]bis[5-(thiophen-2-ylmethylene)2-(p-tolylimino)thiazolidin-4-one]

(2g)

3,3'-[4,4'-Methylenebis(4,1-phenylene)]bis $\{5-[(3-$ methylthiophen-2$\mathrm{yl}$ )methylene $]-2-(\mathrm{p}$-tolylimino $)$ thiazolidin-4-one

(2h) $2.43\left(3 \mathrm{H}, \mathrm{s}, \mathrm{CH}_{3}\right)$,

$3060(=\mathrm{C}-\mathrm{H}), \quad 3.97\left(2 \mathrm{H}, \mathrm{s}, \mathrm{CH}_{2}\right)$,

$2924(\mathrm{C}-\mathrm{H}), \quad 6.92(2 \mathrm{H}, \mathrm{d}, J=8.2 \mathrm{~Hz}, \mathrm{Ar}-\mathrm{H})$

$1687(\mathrm{C}=\mathrm{O}), \quad 6.99(1 \mathrm{H}, \mathrm{d}, J=5.0 \mathrm{~Hz}, \mathrm{Ar}-\mathrm{H})$,

Yellow powder

$198-9$

$1670(\mathrm{C}=\mathrm{N})$,

$1592(\mathrm{C}=\mathrm{C})$,

$1362(\mathrm{C}-\mathrm{N})$

$840(\mathrm{C}-\mathrm{S})$

$7.13(2 \mathrm{H}, \mathrm{d}, J=8.2 \mathrm{~Hz}, \mathrm{Ar}-\mathrm{H})$

$7.46(2 \mathrm{H}, \mathrm{dd}, \mathrm{J}=8.6 ; 1,7 \mathrm{~Hz}, \mathrm{Ar}-\mathrm{H})$,

$7.50(1 \mathrm{H}, \mathrm{d}, J=5.0 \mathrm{~Hz}, \mathrm{Ar}-\mathrm{H})$,

7.54(3H, t, J=7.9;7.4 Hz, Ar-H)

$8.06(1 \mathrm{H}, \mathrm{s}$, vinyl $\mathrm{H})$

$2.41\left(3 \mathrm{H}, \mathrm{s}, \mathrm{CH}_{3}\right)$,

$4.04\left(1 \mathrm{H}, \mathrm{s}, \mathrm{CH}_{2}\right)$

$3024(=\mathrm{C}-\mathrm{H}), \quad 6.88(2 \mathrm{H}$, brd $, J=8.2 \mathrm{~Hz}, \mathrm{Ar}-\mathrm{H})$

$2910 \mathrm{C}-\mathrm{H}), \quad 6.93(1 \mathrm{H}, \mathrm{dd}, J=4.9 ; 4.7 \mathrm{~Hz}, \mathrm{Ar}-\mathrm{H})$,

$1712(\mathrm{C}=\mathrm{O}), \quad 7.14(1 \mathrm{H}, \mathrm{d}, J=4.9 \mathrm{~Hz}, \mathrm{Ar}-\mathrm{H})$,

Light yellow

powder

214-5

$1630(\mathrm{C}=\mathrm{N})$,

$1596(\mathrm{C}=\mathrm{C})$

$1352(\mathrm{C}-\mathrm{N})$

819 (C-S)

7.18 (2H, brd, J=8.4, Ar- $\mathrm{H})$

$21(2 \mathrm{H}$, brd $\mathrm{J}=8.2, \mathrm{Ar}-\mathrm{H})$

$7.40(2 \mathrm{H}$, brd, $J=8.4, \operatorname{Ar}-\mathrm{H})$

$7.53(1 \mathrm{H}, \mathrm{d}, J=4.5, \mathrm{Ar}-\mathrm{H})$,

$8.00(1 \mathrm{H}, \mathrm{s}$, vinyl $\mathrm{H})$

$2.35\left(3 \mathrm{H}, \mathrm{s}, \mathrm{CH}_{3}\right)$,

$3027(=\mathrm{C}-\mathrm{H}), \quad 2.41\left(3 \mathrm{H}, \mathrm{s}, \mathrm{CH}_{3}\right)$,

$2918(\mathrm{C}-\mathrm{H})$

$6.88(2 \mathrm{H}, \mathrm{brd}, \mathrm{J}=8.2 \mathrm{~Hz}, \mathrm{Ar}-\mathrm{H})$,

$1712(\mathrm{C}=\mathrm{O}), \quad 6.94(1 \mathrm{H}, \mathrm{dd}, J=8.5 \mathrm{~Hz}, \mathrm{Ar}-\mathrm{H})$

Yellow powder

207-8

$1630(\mathrm{C}=\mathrm{N})$,

$7.13(1 \mathrm{H}, \mathrm{d}, J=5.0 \mathrm{~Hz}, \mathrm{Ar}-\mathrm{H})$

$1596(\mathrm{C}=\mathrm{C}), \quad 7.19(2 \mathrm{H}$, brd, $J=8.2, \mathrm{Ar}-\mathrm{H})$

$1352(\mathrm{C}-\mathrm{N}), \quad 7.39(2 \mathrm{H}, \mathrm{brd}, \mathrm{J}=8.4, \mathrm{Ar}-\mathrm{H})$

819 (C-S) $\quad 7.46(1 \mathrm{H}, \mathrm{d}, J=4.5, \mathrm{Ar}-\mathrm{H})$

$8.01(1 \mathrm{H}, \mathrm{s}$, vinyl $\mathrm{H})$

$766(\mathrm{M}+1)$

$765\left(\mathrm{M}^{+}\right)$,

$764(M-1)$,

569,

389

314,

242

$766(M+1)$ $765\left(\mathrm{M}^{+}\right)$,

$764(M-1)$,

569 ,

389,

314 ,

242

$794(M+1)$ $793\left(M^{+}\right)$,

$792(M-1)$,

597,

389,

314,

242 


\section{CONCLUSION}

In conclusion, this study reports an appropriate and reliable synthesis of 5-substituted bisiminothiazolidinones (2a-h) starting from previously prepared bis-thioureas (1a-d), chloroacetic acid and substituted thiophene-2carbaldehyde via one-pot green synthesis.

\section{ACKNOWLEDGEMENTS}

Thanks are due to Yıldız Technical University Scientific Research Projects Coordination's support in this study. Project Number: 2013-0102-GEP03.

\section{REFERENCES}

1. Marvaniya HM, Modi KN, Sen DJ. Greener reactions under solvent free conditions. International Journal of Drug Development and Research. 2011;3(2):34-44.

2. Sarkar A, Santra S, Kundu SK, Hajra A, Zyryanov GV, Chupakhin ON, Charushin VN, Majee A. A decade update on solvent and catalyst-free neat organic reactions: A step forward towards sustainability. Green Chemistry. $2016 ; 18: 4475-4525$.

3. Wender PA, Handy ST, Wright DL. Towards the ideal synthesis. Chemistry and Industry. 1997;19:765-768.

4. Verma A, Saraf SK. 4-Thiazolidinone - A biologically active scaffold. European Journal of Medicinal Chemistry. 2008;43(5):897-905.

5 Edwards PJ. Thiazolidinone derivatives targeting drug - resistant lung cancer cells. Drug Discovery Today. 2008;13(23/24):1107-1108.

6. Gududuru V, Hurh E, Dalton JT, Miller DD. Synthesis and antiproliferative activity of 2-aryl4-oxo-thiazolidin-3-yl-amides for prostate cancer. Bioorganic and Medicinal Chemistry Letter. 2004;14: 5289-5293.

7. Liu HL, Li Z, Anthonsen T. Synthesis and fungicidal activity of 2-imino-3-(4-arylthiazol-2$\mathrm{yl}$ )-thiazolidin-4-ones and their 5-arylidene derivatives. Molecules. 2000;5(9):1055-1061.

8. Romine J, Martin S, Snyder L, Serrano-Wu M, Deshpande $M$, Whitehouse $D$, et al. iminothiazolidinones as inhibitors of hcv replication. Princeton, New Jersey; US 2005/0096364A. p. 57.

9. Hu J, Wang Y, Wei X, Wu X, Chen G, Cao G, Shen $X$, Zhang $X$, Tang $Q$, Liang G, Li X. Synthesis and biological evaluation of novel thiazolidinone derivatives as potential anti-inflammatory agents. European Journal of Medicinal Chemistry. 2013;64:292-301.
10. Kücükgüzel $S G$, Oruç $E E$, Rollas $S$, Şahin $F$, Özbek A. Synthesis, characterisation and biological activity of novel 4-thiazolidinones, 1,3,4-oxadiazoles and some related compounds. European Journal of Medicinal Chemistry. 2002;37(3): 197-206.

11. Metwally MA, Farahat AA, Abdel-Wahab BF. 2Amino-4-thiazolidinones: synthesis and reactions. Journal of Sulfur Chemistry. 2010;31(4):315-349.

12. Ottana $R$, Maccari $R$, Barreca ML, Bruno G, Rotondo A, Rossi A, Chiricosta G, Di Paola R, Sautebin L, Cuzzocread S, Vigoritaa MG. 5Arylidene-2-imino-4-thiazolidinones: Design and synthesis of novel anti-inflammatory agents. Bioorganic and Medicinal Chemistry. 2005; 13:4243-4252.

13. Chavan AA, Pai NR. Synthesis and antimicrobial screening of 5-arylidene-2-imino-4thiazolidinones. Arkıvoc. 2007;xvi:148-155.

14. Zhou JF, Sun XJ, Zhu FX, Li YL, Gong GX. A Facile Synthesis of 5-Arylidene-2-imino-4thiazolidinones under microwave irradiation. Synthetic Communications. 2008;38:4182-4187.

15. Khazaei A, Veisi $H$, Safaei $M$, Ahmadian $H$. Green synthesis of 5-arylidene-2,4thiazolidinedione, 5-benzylidene rhodanine and dihydrothiophene derivatives catalyzed by hydrated ionic liquid tetrabutylammonium hydroxide in aqueous medium. Journal of Sulfur Chemistr. 2014;35(3):270-278.

16. Abbas N, Zaib S, Bakht SM, Ibrar A, Khan I, Batool S, Saeed A, Iqbal J. Symmetrical aryl linked bis-iminothiazolidinones as new chemical entities for the inhibition of monoamine oxidases: Synthesis, in vitro biological evaluation and molecular modelling analysis. Bioorganic Chemistry. 2017;70:17-26.

17. Fakhar I, Yamin BM, Hasbullah SA. Synthesis and characterization of bis-thiourea having amino acid derivatives. American Institute of Physics Conference Proceedings, 2016;1784, 030012.

18. Masaki M, Kitahara T, Kurita H, Ohta M. A New Method for the Removal of Chloroacetyl Groups. Journal of the American Chemical Society. $1968 ; 90: 4508-4509$.

19. Yella R, Ghosh H, Patel BK. It is "2-imino-4thiazolidinones" and not thiohydantoins as the reaction product of 1,3-disubstituted thioureas and chloroacetylchloride. Green Chemistry. 2008; 10:1307-1312.

20. Bhattacharjee SK, Mukherjee R, Hazarika D, Chakraborty A, Acharjee SR. Synthesis of bioactive organosulfur compounds from phenyl isothiocyanate (PITC). Acta Ciencia Indica Chemistry. 2007;33(3):295-302. 
21. Zhang LP, Shang XB, Wu QF, Zhang Y, Li JP. Highly efficient method for the synthesis of 1,4phenylenedithioureas under solvent- and catalyst-free conditions promoted by microwave irradiation. Synthetic Communications. 2012;42(7),1045-1052.
22. Pandya D, Nair KB. Bridged bis(4thiazolidinones) and related compounds with antibacterial $1993 ; 48(6): 414-17$. 